\title{
Superconductivity in Polycrystalline Pressed Samples of Organic Metals
}

\author{
D. Schweitzer*, S. Gärtner, H. Grimm, E. Gogu and H. J. Keller* \\ Max Planck Institut für Medizinische Forschung, AG Molekulkristalle and *Anorganisch \\ Chemisches Institut der Universität Heldelberg, Heidelberg, Federal Republic of Germany \\ (Received 1 December 1988 by Manuel Cardona)
}

\begin{abstract}
We report bulk superconductivity at ambient pressure in polycrystalline pressed samples of $\alpha_{t}-(\text { BEDT - TTF })_{2} I_{3}$. As in single crystals of $\alpha_{t}-(\text { BEDT - TTF })_{2} I_{3}$ the onset for superconductivity in the polycrystalline pressed samples lies at about $9 \mathrm{~K}$ but the transition is broader. Zero resistivity is observed at $2.2 \mathrm{~K}$ and the middle of the transition is found at $5 \mathrm{~K}$. The measurement of the ac susceptibility shows that at $2 \mathrm{~K}$ about $50 \mathrm{x}$ of the sample is superconducting, but at this temperature the transition is still not saturated. The observation of bulk superconductivity in this polycrystalline pressed samples is of special interest because it demonstrates that organic superconductors can be used in principle as well for the production of electronic devices such as squids and superconducting cables. In addition the finding of bulk superconductivity in large pressed samples of crystallites of the typical diameter of $1 \mu \mathrm{m}$ of an organic metal indicates that the observation of superconductivity in organic polymers should be possible as well.
\end{abstract}

Recently bulk superconductivity at $8 \mathrm{~K}$ and ambient pressure was reported in single crystals of the quasi two dimensional organic metal $\alpha_{t}-(\text { BEDT-TTF })_{2} I_{3}$ [1]. From measurements of the upper critical fields $\mathrm{H}_{\mathrm{C} 2}$ and using an anisotropic effective mass model in the picture of the Ginzburg Landau theory the coherence lengths at $0 \mathrm{~K}$ of $\xi_{\lrcorner}=22 \mathrm{~A}, \xi_{\|}=120 \mathrm{~A}$ and the penetration depths of $\lambda_{1}=750 \mathrm{~A}, \lambda_{11}=4300 \mathrm{~A}$ were obtained [2]. These results led to the idea that, similar to the high temperature superconductors of the metal oxides, it should be possible to obtain bulk superconductivity in polycrystalline pressed samples of $\alpha_{t}-(\text { BEDT }- \text { TTF })_{2} l_{3}$. The remark able fact of such finding would be that in such a case organic superconductors in principle could be used as well for the preparation of electronic devices such as squids and might even be suitable for the preparation of superconducting cables. Such a finding would further be an indication that superconductivity should be observable as well in organic polymers.

Samples of the size $4 \times 1 \times 0.5 \mathrm{~mm}$ were prepared from grained single crystals (the resulting crystallites from the graining process had typical diameters of $0.5-10$ $\mu \mathrm{m})$ of the organic metal $\alpha-\left(\right.$ BEDT -TTF) ${ }_{2} I_{3}[3,4]$ by applying a pressure of $(3-10) \cdot 10^{3} \mathrm{~kg} / \mathrm{cm}^{2}$ to the powder. These mechanically very stable samples were tempered at $75^{\circ} \mathrm{C}$ for 50 hours in order to obtain samples of $\alpha_{t}-\left(\right.$ BEDT - TTF ) ${ }_{2} I_{3}$ [1]. The conductivity was measured with the usual four point method.

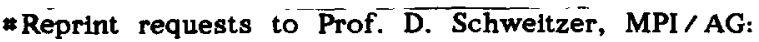
Molekülkristalle, Jahnstr. 29, 6900 Heidelberg, FRG
The room temperature conductivity of these polycrystalline samples is $15(\Omega \mathrm{cm})^{-1}$ Figure 1 shows the temperature dependence of the resistivity of the polycrystalline sample (note the double logarithmic scale). By cooling the sample down from room temperature the resistivity decreases as in a metal. The metallic character was confirmed by ESR measurements, where in the temperature range $10-300 \mathrm{~K}$ a more or less temperature independent spin susceptibility was observed. The absolute value of the susceptibility is $+(4.6 \pm$ $0.25) \times 10^{-4} \mathrm{emu} / \mathrm{mole}$, a value which agrees well with the one obtained for the crystals of $\alpha_{t}-\left(\right.$ BEDT-TTF ${ }_{2} I_{3}$ [5].

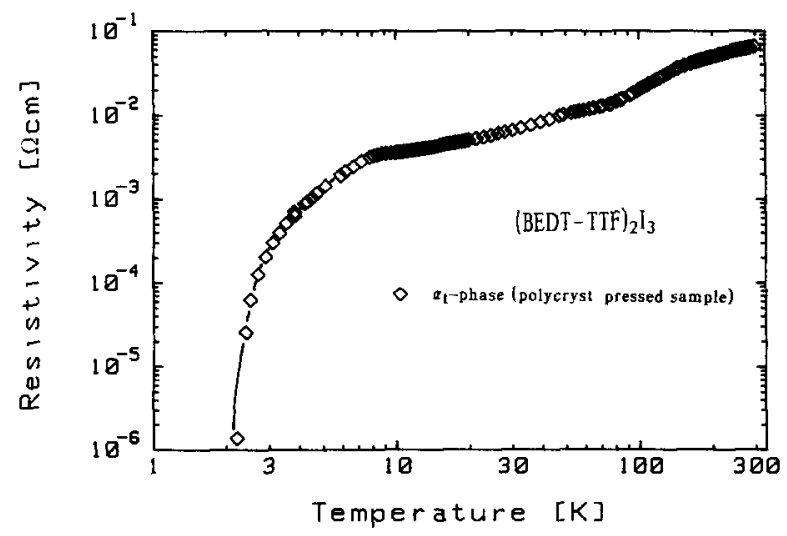

Eigure 1: Resistivity versus temperature (double logarithmic scale) of a polycrystalline pressed sample of $\alpha_{t}-(\text { BEDT }- \text { TTF })_{2} I_{3}$. 
Figure 2 shows the resistivity (normalized at $12 \mathrm{~K}$ ) of the polycrystalline sample and of a crystal of $\alpha_{t}$ (BEDT - TTF) ${ }_{2} I_{3}$ in the temperature range below $12 \mathrm{~K}$. In both samples the onset of superconductivity in the resisttvity is found near $9 \mathrm{~K}$. In contrast to the crystal of $\alpha_{t}$-(BEDT-TTF $)_{2} I_{3}$ the superconducting transition in the polycrystalline sample is much broader. Zero resistivity (within the experimental facilities) in the polycrystalline sample is found at $2.2 \mathrm{~K}$, while in the case of the crystals of $\alpha_{t}-(\text { BEDT }-T T F)_{2} I_{3}$ zero resistivity appears already at $6 \mathrm{~K}$.

In order to obtain information whether the superconductivity observed in the resistivity is a bulk effect of the sample or not we measured the ac susceptibility at a frequency of $3 \mathrm{MHz}$ with a fleld of about 0.2 Gauss. Figure 3 shows the increase in resonance frequency of the LC-circuit due to exclusion of the $\mathrm{rf}$ - fleld by diamagnetic shielding currents in the polycrystalline sample of $\alpha_{t}-(\text { BEDT }- \text { TTF })_{2} I_{3}$. There is a clear evidence of an onset of diamagnetic shielding below $7 \mathrm{~K}$. This behaviour is in contrast to the observations usually made in single crystals of organic superconductors, where the temperature for the onset of diamagnetic shielding is at the the temperature where the resistivity reaches zero. Here in the polycrystalline sample the onset for the diamagnetic shielding is far above the temperature where the resistivity becomes zero. The signal (see Fig. 3) which still increases on cooling to $2 \mathrm{~K}$ corresponds to about $50 \%$ with respect to that expected for a perfect superconductor, indicating a clear bulk effect of the superconductivity in the polycrystalline sample of $\alpha_{t}-(B E D T-T T F)_{2} I_{3}$. Nevertheless, the large temperature range in which the frequency shift of the resonance frequency is observed shows that an inhomogenous distribution of superconducting transitions exist in the sample. A similar broad distribution was already observed in the case of the crystals of $\alpha_{t}-(\text { BEDT - TTF })_{2} I_{3}$ and might be due to the annealing procedure during the preparation of the $\alpha_{t}$-phase.

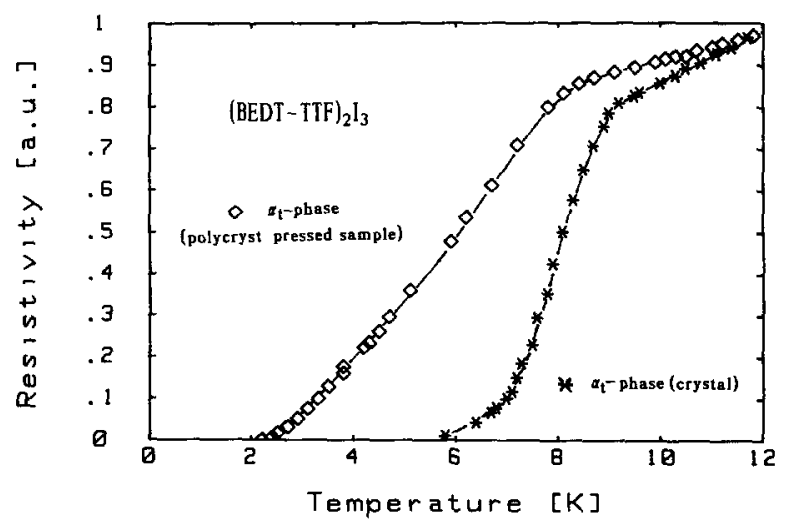

Figure 2: Resistivity (nórmalized at $\overline{12} \overline{\mathrm{K}}$ ) of a polycrystalline sample of $\alpha_{t}-(\text { BEDT }-T T F)_{2} I_{3}$ as well as of a crystal of $\alpha_{t}-(\text { BEDT }- \text { TTF })_{2} I_{3}$ in the temperature range below $12 \mathrm{~K}$.

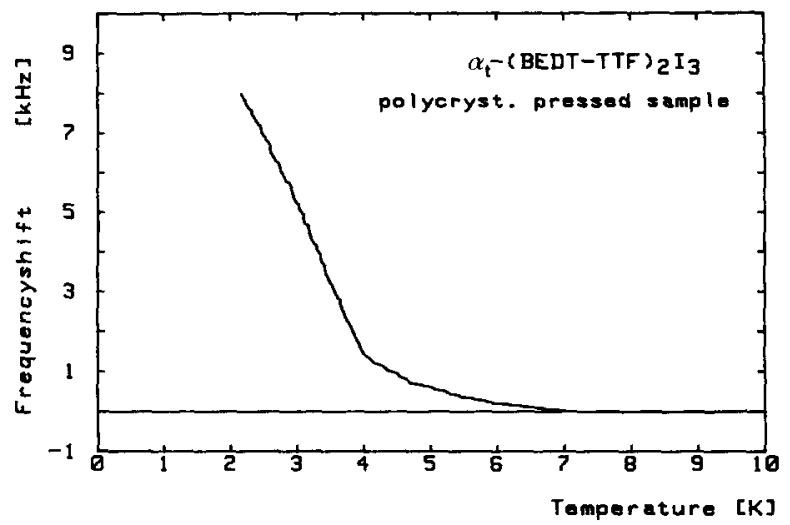

Eigure 3: Increase of the resonance frequency of an LC-circuit ( $3 \mathrm{MHz})$ due to exclusion of the rf-fleld by diamagnetic shielding currents (ac susceptibility) in a polycrystalline pressed sample of $\alpha_{t}-(\text { BEDT }- \text { TTF })_{2} I_{3}$ by cooling the sample from $10 \mathrm{~K}$ down to $2 \mathrm{~K}$.

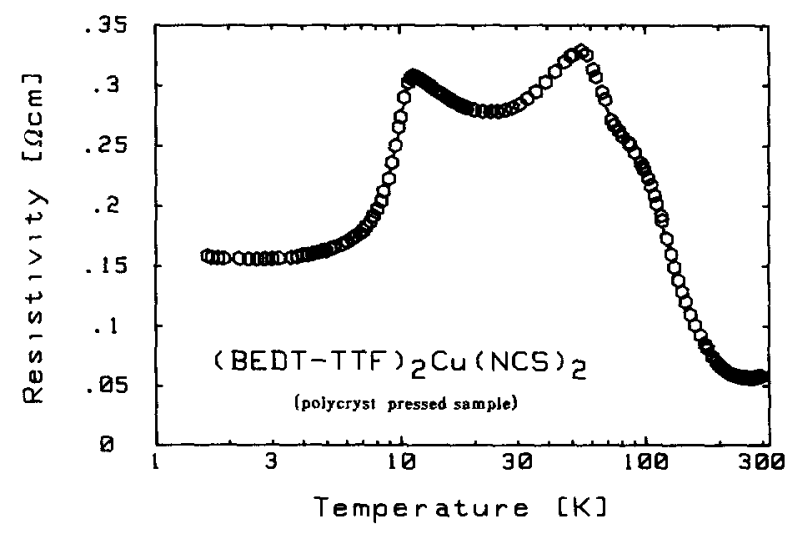

Figure 4: Resistivity versus temperature of a polycrystalline sample of $(\mathrm{BEDT}-\mathrm{TTF})_{2} \mathrm{Cu}(\mathrm{NCS})_{2}$.

It should be mentioned that the preparation of pressed polycrystalline samples by starting already with powdered crystals of $\alpha_{t}-(\text { BEDT }- \text { TTF })_{2} I_{3}$ does not lead to superconductivity in the samples. This behavior is due to a phase transition of the $\alpha_{\mathrm{t}}$-phase under the applied pressure during the sample preparation as will be reported elsewhere [6]. In order to obtain superconductivity in the polycrystalline samples the preparation has to be started with powdered crystals of $\alpha-(\text { BEDT }- \text { TTF })_{2} I_{3}$ and the pressed samples have to be annealed. Samples prepared in this manner should not be pressed again.

We have further prepared pressed samples of polycrystalline (BEDT-TTF) ${ }_{2} \mathrm{Cu}(\mathrm{NCS})_{2}$. In the single crystals of (BEDT - TTF ${ }_{2} \mathrm{Cu}\left(\mathrm{NCS}_{2}\right)_{2}$ a relatively sharp superconducting transition at $10.4 \mathrm{~K}$ exists $[7,8]$. The transition temperature of $10.4 \mathrm{~K}$ is the highest observed $T_{c}$ among the organic superconductors at the moment. In addition the superconductivity in the single crystals of (BEDT-TTF) ${ }_{2} \mathrm{Cu}(\mathrm{NCS})_{2}$ is nearly a $100 \mathrm{x}$ bulk effect $[8,9]$. 
The room temperature conductivity of the pressed polycrystalline samples of (BEDT-TTF) ${ }_{2} \mathrm{Cu}(\mathrm{NCS})_{2}$ is with $15(\Omega \mathrm{cm})^{-1}$ only a little smaller compared to the values of $20-60(\Omega \mathrm{cm})^{-1}$ observed in single crystals of (BEDT - TTF $)_{2} \mathrm{Cu}(\mathrm{NCS})_{2}$. Figure 4 shows the temperature dependence of the resistivity of a polycrystalline pressed sample (size as above). Besides the fact, that all the phase transitions observed before in the single crystals [8] can be found now in the resistivity characteristic of the polycrystalline sample much more pronounced, the resistivity starts again to decrease below $11 \mathrm{~K}$, the onset temperature for superconductivity in

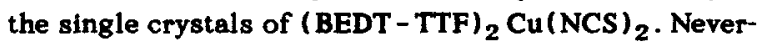
theless, here in the polycrystalline sample the resistivity does not become zero, in fact at $1.3 \mathrm{~K}$ the resistivity is still larger than at room temperature. The measurement of the ac susceptibility in the polycrystalline sample did show an onset of diamagnetic shielding below
$10 \mathrm{~K}$, but at $2 \mathrm{~K}$ less than $1 \mathrm{X}$ of the sample was affected. In the moment it is not clear why the polycrystalline sample does not show bulk superconductivity as the single crystals. It might be, that due to the pulverization of the crystals of (BEDT-TTF) ${ }_{2} \mathrm{Cu}(\mathrm{NCS})_{2}$ or due to the pressure during the preparation of the sample paramagnetic centers are produced and therefore the superconductivity is suppressed.

In conclusion, bulk superconductivity at amblent pressure exists in polycrystalline pressed samples of $\alpha_{t}-(\text { BEDT - TTF })_{2} I_{3}$, while in the polycrystalline samples of (BEDT-TTF) ${ }_{2} \mathrm{Cu}(\mathrm{NCS})_{2}$ such a bulk effect could not be observed.

The construction of a squid from polycrystalline pressed $\alpha_{t}$-(BEDT-TTF $)_{2} I_{3}$ is under progress.

We gratefully acknowledge financial support of this work by Deutsche Forschungsgemeinschaft.

\section{REFERENCES}

1. D. Schweitzer, P. Bele, H. Brunner, E. Gogu, U. Haeberlen, I. Hennig, T. Klutz, R. Swietlik, H. J. Keller; Z. Phys. B-Condensed Matter 67, 489 (1987).

2. E. Gogu, D. Schweitzer and H. J. Keller; Physica C 153-155, 491 (1988).

3. K. Bender, K. Dietz, H. Endres, H. W. Helberg, I. Hennig, H. J. Keller, H. W. Schäfer, D. Schwettzer; Mol. Cryst, Liq. Cryst. 107, 45 (1984).

4. K. Bender, I. Hennig, D. Schweitzer, K. Dietz, H. Endres and H. J. Keller; Mol. Cryst. Liq. Cryst. 108, 359 (1984).
5. S. Klotz, J. S. Schilling, S. Gärtner, D. Schweltzer; Solid State Comm. 67, 981 (1988).

6. R. Zamboni, D. Schweitzer and H. J. Keller; to be published.

7. H. Urayama, H. Yamochi, G. Saito, K. Nozawa, T. Sugano, M. Kinoshita, S. Saito, K. Oshima, A. Kawamoto and J. Tanaka; Chem. Lett. 1988, 55 (1988).

8. S. Gärtner, E. Gogu, I. Heinen, H. J. Keller, T. Klutz, D. Schweitzer; Solid State Comm. 6S, 1531 (1988).

9. K. Nozawa, T. Sugano, H. Urayama, H. Yamochi, G. Saito and M. Kinoshita; Chem. Lett. 1988, 617 (1988). 